\title{
The cytokine profile of women with severe anxiety and depression during pregnancy
}

\author{
P. Leff Gelman ${ }^{1 *}$, I. Mancilla-Herrera ${ }^{3}$, M. Flores-Ramos ${ }^{2,6}$, M. F. Saravia Takashima ${ }^{3}$, F. M. Cruz Coronel ${ }^{3}$, \\ C. Cruz Fuentes ${ }^{2}$, A. Pérez Molina², J. Hernández-Ruiz ${ }^{4}$, F. S. Silva-Aguilera ${ }^{4}$, B. Farfan-Labonne', \\ D. Chinchilla-Ochoa', S. Garza Morales ${ }^{1}$ and I. Camacho-Arroyo ${ }^{5}$
}

\begin{abstract}
Background: Controversial findings regarding the association between pro-inflammatory cytokines and depression have been reported in pregnant subjects. Scarce data about anxiety and its relationships with cytokines are available in pregnant women. To understand the association between anxiety and cytokines during pregnancy, we conducted the present study in women with or without depression.

Methods: Women exhibiting severe depression (SD) and severe anxiety (SA) during the 3rd trimester of pregnancy $(n=139)$ and control subjects exhibiting neither depression nor anxiety $(n=40)$ were assessed through the Hamilton Depression Rating Scale (HDRS) and the Hamilton Anxiety Rating Scale (HARS). Serum cytokines were measured by a multiplex bead-based assay. Correlation tests were used to analyze the data and comparisons between groups were performed. A general linear model of analysis of variance was constructed using the group as a dependent variable, interleukin concentrations as independent variables, and HDRS/HARS scores and gestational weeks as covariables.

Results: The highest levels of Th1- (IL-6, TNF-a, IL-2, IFN- $)$, Th17- (IL-17A, IL-22), and Th2- (IL-9, IL-10, and IL-13) related cytokines were observed in women with SD + SA. The SA group showed higher concentrations of Th1- (IL-6, TNF-a, IL2, IFN- $\gamma$ ) and Th2- (IL-4, and IL-10) related cytokines than the controls. Positive correlations were found between HDRS and IL-2, IL-6, and TNF- $a$ in the SA group $(p<0.03)$, and between HDRS and Th1- (IL-2, IL-6, TNF-a), Th2- (IL-9, IL-10, IL13) and Th17- (IL-17A) cytokines $(p<0.05)$ in the SD + SA group. After controlling the correlation analysis by gestational weeks, the correlations that remained significant were: HDRS and IL-2, IL-6, IL-9, and IL-17A in the SD + SA group $(p<0.03)$. HARS scores correlated with IL-17A in the SA group and with IL-17A, IL-17F, and IL-2 in the SD + SA group $(p<0.02)$. The linear model of analysis of variance showed that HDRS and HARS scores influenced cytokine concentrations; only IL-6 and TNF-a could be explained by the group.
\end{abstract}

Conclusions: We found that the cytokine profiles differ when comparing pregnant subjects exhibiting SA with comorbid SD against those showing only SA without depression.

Keywords: Cytokines, Inflammation, Pregnancy, Depression, Anxiety

\footnotetext{
* Correspondence: pleff@ymail.com

${ }^{1}$ Instituto Nacional de Pernatologia, Isidro Espinosa de los Reyes, Montes

Urales \# 800, Col Lomas de Virreyes, 11000 (Mexico City), CD MX, Mexico

Full list of author information is available at the end of the article
}

(c) The Author(s). 2019 Open Access This article is distributed under the terms of the Creative Commons Attribution 4.0 International License (http://creativecommons.org/licenses/by/4.0/), which permits unrestricted use, distribution, and reproduction in any medium, provided you give appropriate credit to the original author(s) and the source, provide a link to the Creative Commons license, and indicate if changes were made. The Creative Commons Public Domain Dedication waiver (http://creativecommons.org/publicdomain/zero/1.0/) applies to the data made available in this article, unless otherwise stated. 


\section{Background}

Major depressive disorder (MDD) is an important public health issue [1-3] that has been predicted to become the second leading cause of disability by the year 2020 (behind only ischemic heart disease) [4]. Compared to men, women are more than twice as susceptible to depression [5]. Depression during pregnancy is an important topic, both for understanding the pathophysiology of the disease and determining adequate treatment.

The prevalence of perinatal depression differs throughout pregnancy, from $7.4 \%$ in the first, $12.8 \%$ in the second, and $12.0 \%$ in the third trimester $(20.4 \%$ considering the entire pregnancy) [5-7]. Nonetheless, other reports have shown that $\sim 9 \%$ of women suffer major or minor depression during each of the trimesters, while postpartum depression has a prevalence of 13\% (at 3 months postpartum) $[8,9]$. Pregnant women experience more stressful events than non-pregnant women $[10,11]$, and frequently report tiredness or fatigue (87.2-96.5\%) [12], symptoms commonly associated with depression and anxiety [13]. Interestingly, pregnant subjects with antenatal depression have a higher rate of preeclampsia and preterm birth associated with poor fetal and infant outcomes (motor and mental development) [10, 14].

It is well-known that Th1/Th2 and Th17/Treg immune balances are needed to maintain a successful pregnancy [5] and thereby of the conceptus [15]. Recent studies have shown that pregnant patients with MDD display a preferential inflammatory response $[16,17]$ and higher levels of circulating steroids (i.e., cortisol) when compared to healthy pregnant women [18].

Furthermore, human and animal studies have provided clear evidence that chronic activation of microglial cells [19-21] triggers mood disorders [22] through the release of a variety of neurotoxins, pro-inflammatory cytokines, free radicals, nitric oxide, chemokines, proteinases, and eicosanoids [19], causing neuronal dysfunction and/or aggravating underlying pathologies [23].

Several pieces of evidence support the premise that chronic psychological distress contributes to adverse pregnancy outcome, such as preterm birth, proposing that complex interactions among stress, individual health behaviors, genomic background, and the physiology of pregnancy itself might determine both maternal and child outcomes [24-26]. Such interactions involve immune mediators, such as interleukins (IL)-1 $\beta$, IL-6, IL-10, TNF- $\alpha$, hormones, and several other compounds (i.e., prostaglandins, chemokines) $[5,27]$.

With regard to mood-related disorders during pregnancy, multiple studies shed controversial results between depressive scores (CES-D, EPDS) and blood levels of Th1- and Th2-related biomarkers in women exhibiting MDD during mid and late pregnancy and the early postpartum period [28-30]. In line with this, one study found positive correlations between depressive scores and circulating levels of IL- 6 and TNF- $\alpha$ [28]; whereas other studies have reported non-significant associations between Th1-related (IL)-6) and Th2-related (IL)-10) biomarkers and depressive symptoms during pregnancy [29]. However, this latter study reported that depressive symptoms during pregnancy, as well as the IL6 and IL-10 biomarkers, were significant predictors of postpartum Edinburgh Perinatal Depression Scale (EPDS) score [29].

A similar study focused on exploring the relationship between prenatal psychological symptoms, prenatal stress, and the increased risk for atopic diseases in the offspring found positive correlations between concentrations of potentially proallergenic cytokines (IL-5, IL-13) and maternal prenatal psychological symptoms [30]. This study also showed that a higher (IFN)- $\gamma /$ IL-4 ratio positively correlated with the depressive score; whereas IL-12 and IL-13 correlated with pregnancy-related anxiety score; besides, IL-9 and IL-13 displayed significant correlations with mood-related symptoms in women exhibiting depression and anxiety from early to mid-gestation [30].

An interesting study demonstrated positive correlations between Th1 (IL-6) and Th2 (IL-10) cytokines with depression, anxiety, and perceived stress in women during mid-pregnancy [14-28 gestation weeks (gwks)]. Moreover, the cited study showed that plasma levels of IL1- $\beta$, IL-6, and IL-10 were significantly lower among women who took selective serotonin reuptake inhibitors (SSRI) during pregnancy [27]. This study provides evidence that major depression and severe anxiety during pregnancy significantly contribute to immune system pathways, thereby enhancing the risk of a poor pregnancy with negative obstetric outcomes, as previously reported [5].

Despite a large number of studies exploring the associations between depression and inflammation [31], little is known about the relationship between anxiety and inflammation during pregnancy [32]. Recent studies have shown that several physiological/biochemical variables are associated with prenatal anxiety, such as polyunsaturated fatty acids, elevated cortisol, low levels of the enzyme that metabolizes cortisol, and elevated pro-inflammatory cytokines (IL1, IL-6, TNF- $\alpha$ ) [33]. Thus, despite the associations between inflammatory biomarkers and depressive disorders during pregnancy, the link between immune mediators and anxiety remains unclear.

In such a context, we formulated the following hypothesis: a) pregnant women exhibiting severe anxiety with or without comorbid depression should display a significant increase in distinct immune mediators in blood when compared to healthy pregnant women; b) the increase in Th1 and/or Th2-immune mediators should display significant correlations with anxiety and depressive symptoms, reflecting the influence of affective 
disorders in the dysregulation of the maternal immune system and immune balance (Th1:Th2) in pregnancy.

\section{Methods \\ Design of the Study}

We conducted a transversal study at the National Institute of Perinatology (Neuroscience Department, Mexico City) together with the Department of Gynecology and Obstetrics (GO) at the General Hospital of Mexico (HGM, Dr. Eduardo Liceaga, Mexico City) from October 2014 to December 2016. Approval from the Institution Ethical Committee was obtained before the beginning of the study (HGM, D1/14/112/04/072, 2014-2016). Written informed consent was obtained from all women recruited before the study began. Pregnant women were admitted at the Prenatal Control Outpatient Unit of the GO Department during the second and third trimester of gestation. Clinical, sociodemographic, obstetric, and psychiatric characteristics of the recruited participants were recorded into a database. At entry, participants in the study required first, second, and third-trimester lab tests (blood count, biochemical testing, urinalysis, thyroid function), 2D fetal ultrasound, and Doppler Monitoring, including lab test for sex hormones, cortisol, and DHEA-S (data not shown). All patients were inhabitants of Mexico City and surrounding areas.

\section{Participants}

Patients recruited to the study attended the Prenatal Control Outpatient Unit. Women between 16- and 30-years-old, exhibiting a pregnancy between 20 and 39 gwks, were invited to participate in the study. In their first visit, a complete clinical evaluation was carried out, including anthropometric evaluation. Participants were asked to complete the self-reported questionnaires used to measure anxiety and depressive symptoms; in the same interview, an evaluation of socio-demographic variables was done, including marital status, education level, and working status. Patients exhibiting a cutoff score > 15 in the Hamilton Anxiety rating scale (HARS) were considered as having severe anxiety. Patients with a cutoff score as $<5$ were included in the control group. We excluded patients who were receiving psychotropic medication, patients with illicit substance use, previous psychiatric diagnosis, obstetric pathologies (diabetes, hypertension, preeclampsia), infections, and medical diseases (past and present neurological, metabolic, cardiovascular, degenerative, and rheumatic disorders). Upon completion of the questionnaires, participants displaying high-rating scores for moderate to severe anxiety and/or depressive symptoms were remitted to a psychiatric outpatient service for mood-disorder management. After psychiatry clinical interview and completion of the questionnaires, patients were remitted to the GO Department for blood extraction and analysis of their serum cytokine profile.

We assessed a total of 298 pregnant women: patients, exhibiting low to high levels of depressive symptoms and comorbid anxiety $(n=141)$, pregnant women exhibiting anxiety symptoms without depression $(n=100)$ mostly during the 3rd trimester of pregnancy (28-40 gwks), and healthy pregnant women displaying no affective disorders $(n=57)$. All participants were screened for depressive symptoms and anxiety, serum cytokines and sociodemographic parameters. Some of the participants were excluded due to incomplete questionnaires, absence or incomplete laboratory tests, or inconsistencies in the evaluation of both depressive and anxiety symptoms. Our present work included three groups of participants a) patients exhibiting severe anxiety without depression (SA, $n=72$ ); b) patients exhibiting severe anxiety and comorbid severe depression (SA $+\mathrm{SD}, n=67)$; and c) healthy pregnant women as controls (CTRL, $n=40)$.

\section{Criteria and evaluation instruments}

Criteria considered for patient recruitment included the presence of severe anxiety according to the 14 item-Hamilton Anxiety Rating Scale (HARS) [34], which assesses the severity of anxiety symptoms with a cutoff score $>15$ for severe anxiety. Depressive symptoms were evaluated using the 17-Item Hamilton Depression Rating Scale (HDRS), with a cutoff score $>19$ for major depressive disorder [35, 36]; and evaluated by psychiatric clinical interview following the Diagnostic and Statistical Manual of Mental Disorders (DSM-5; American Psychiatric Association, 2015). HDRS and HARS were applied to healthy women to confirm the absence of depressive and anxiety symptoms.

Both instruments have been validated in the local language $[36,37]$ and have been demonstrated to be reliable, specific, and sensitive [38]. Rating scores obtained from each instrument were checked by the Psychiatric Department, and final evaluations were recorded in a clinical database. Patients exhibiting an HDRS score $>19$ and HARS score $>17$ were referred to the psychiatry department for further evaluation and treatment.

\section{Blood sampling}

Blood sampling was carried out during daylight under aseptic conditions, and $5.0 \mathrm{~mL}$ of venous blood was collected into sterile $13 \times 100 /$ Vacutainer BD Hemogard Tubes, containing Clot Activator/Polymer Gel for serum separation (Becton \& Dickinson 367,977, USA). Tubes were allowed to clot for $1 \mathrm{~h}$ at $4{ }^{\circ} \mathrm{C}$ before serum separation. Fresh serum was collected by centrifuging samples at $1600 \times \mathrm{G}$ for $10 \mathrm{~min}$, aliquoted into $1.5-\mathrm{mL}$ Eppendorf tubes. and stored at $-70{ }^{\circ} \mathrm{C}$ until further use. 


\section{Quantification of serum cytokine profile}

Serum cytokines (IFN- $\gamma$, TNF- $\alpha$, IL-6, IL-2, IL-5, IL-13, IL-4, IL-10, IL-9, IL-17A, IL-17F, IL-21 and IL-22) were determined by an immunoassay based on multiplex bead array using the LEGENDplex ${ }^{\mathrm{Tm}}$ Human Th Cytokine Panel (Cat. 740,001, BioLegend, USA) following the manufacturer's protocol. Briefly, serum samples $(50 \mu \mathrm{L})$ were incubated with specific IL-antibody coated beads in a mix buffer at room temperature (RT) for $1.5 \mathrm{~h}$. An antigen-antibody reaction was carried out at RT for $1.5 \mathrm{~h}$ in the presence of specific fluorochrome per cytokine. After washes, samples were analyzed in a FACS Aria III flow cytometer (BD, USA). The concentration of analytes was calculated using the LEGENDplexTM Data Analysis Software v 7.0 (Biolegend, CA, USA). The limit of detection (LD) values detected for Th1 cytokines (IL-6, TNF- $\alpha$, IL-2, IFN- $\gamma$ ) were $12.63 \mathrm{pg} / \mathrm{ml}, 78.13 \mathrm{pg} /$ $\mathrm{ml}$, and $9.88 \mathrm{pg} / \mathrm{ml}$, respectively.

The LD values of Th17-related (IL-17A, IL-21, and IL-22) cytokines were $78.13 \mathrm{pg} / \mathrm{ml}$, whereas the LD value of IL-17F was $14.1 \mathrm{pg} / \mathrm{ml}$ respectively; the LD values detected for Th2 cytokines (IL-4, IL-5, IL-13, IL-10, IL-9) were $78.13 \mathrm{pg} / \mathrm{ml}, 9.25 \mathrm{pg} / \mathrm{ml}, 11.68 \mathrm{pg} / \mathrm{ml}, 88.97 \mathrm{pg} / \mathrm{ml}$ and $6.77 \mathrm{pg} / \mathrm{ml}$ respectively.

\section{Statistical analysis}

Cytokine concentrations were presented as mean \pm SEM. Demographic parameters were expressed as mean $\pm \mathrm{SD}$. Bivariate analyses, as well as parametric and non-parametric $\mathrm{t}$-tests, were conducted to assess the associations among immune mediators, demographic parameters, and psychological distress scores. The non-parametric t-test analysis with Welch's correction was used to detect significant differences between the demographic measures and groups, as well as Th-related cytokines among the tested groups. Partial correlation analysis was used to detect significant differences between serum cytokines and demographic parameters, after controlling specific parameters in the studied groups. A post hoc Tukey test analysis was performed to estimate the differences between cytokines and demographic parameters among groups. Also, a general linear model of analysis of variance was constructed using the group as a dependent variable, interleukin concentrations as independent variables, and HDRS/HARS scores and GWKS were introduced as covariables. Statistical analyses were performed using GraphPad Prism 7 (GraphPad Softwares Inc. USA) and SPSS software v.24.0 (Armonk, NY: IBM Corp). For all the statistical analysis, the $p$-value $<0.05$ was considered significant.

\section{Results}

Demographic characteristics

Table 1 shows the sociodemographic characteristics of participants $(n=179)$. Participants were predominantly
Latin women in the 3rd trimester of pregnancy with a gestation week average of 36.4 (range, 20.5-41.1 gwks) and an average age of 26.2 (range $16-39$ years).

\section{Serum cytokine concentrations}

Figures 1, 2, 3 depict the $\log _{10}$ serum concentrations of Th1, Th2, and Th17-related cytokines in the studied groups. As shown, higher concentrations of proinflammatory cytokines (IL-2, IL-6, TNF- $\alpha$, IFN- $\gamma$ ) were observed in the SA + SD group, as compared with the values detected in the SA and the CTRL groups (Fig. 1).

Similarly, higher concentrations of anti-inflammatory (Th2)-related cytokines (IL-4, IL-5, IL-13, IL-10, IL-9) were observed in the SA+SD group, as compared with the values found either in the SA or the CTRL group (Fig. 2).

Furthermore, higher concentrations of (Th17)-related cytokines (IL-17A, IL-17F, IL-21, IL-22) were found in the $\mathrm{SA}+\mathrm{SD}$ group, when compared to the values displayed by the SA group, or between the IL-17A and IL-17F concentrations and those observed in the CTRL group. The highest levels of IL-17A and IL-22 were detected in SA + SD (Fig. 3).

\section{Correlations between serum cytokine concentrations and demographic measures}

Table 2 describes the correlations found between the serum cytokine profile and demographic parameters. As shown, the SA + SD group showed more correlations between cytokines concentrations and studied variables when compared to the SA and CTRL groups. The SA + SD displayed positive correlations between Th1-related (IL)-2, (IL)-6, and TNF- $\alpha$ cytokines and the depression score (HDRS), including the Th2-related (IL)-13, (IL)-10, and (IL)-9 mediators and Th17-associated (IL)-17A cytokines, respectively. The SA group showed similar positive correlations between Th1-related (IL)-2, (IL)-6, and TNF- $\alpha$ cytokines and the HDRS score. Note, however, that only IFN- $\gamma$ correlated either positively or negatively with the anxiety (HARS) score in the SA +SD and the CTRL groups, respectively. Significant differences were observed between cytokines and both HDRS (Tukey test, $p \leq 0.05$ ) and HARS scores (Tukey test, $p \leq 0.03$ ) in both SA + SD and the SA groups, respectively (data not shown).

\section{Correlations between cytokine concentrations and age, anxiety and depressive scores adjusted by gestation weeks}

Table 3 depicts the correlations between the Th-related cytokines concentration and demographic measures when controlling by gestation weeks. As shown, after controlling for this variable, the independent Th-related variables, such as Th1-(IL-2, IL-6), Th2-(IL-9), and Th17-(17A, 17F)-related cytokines positively correlated with both HDRS and HARS scores in both the SA + SD and SA groups, respectively. Furthermore, negative 
Table 1 The non-parametric, t-test with Welch's correction was used to detect statistical differences between demographic measures among the studied groups

\begin{tabular}{|c|c|c|c|}
\hline PARTICPANTS & CTRL & SA & $S A+S D$ \\
\hline$n=179$ & $n=40$ & $n=72$ & $n=67$ \\
\hline & \multicolumn{3}{|c|}{ mean (SD) } \\
\hline AGE (YEAR) & $28.9(6.9)$ & $28.5(8.3)$ & $25.0(6.3)^{*}$ \\
\hline BMI (kg/m2) & $28.2(2.6)$ & $27.9(2.5)$ & $27.7(3.1)$ \\
\hline WEIGHT (kg) & $68.3(7.2)$ & $67.6(6.8)$ & $65.3(12.2)$ \\
\hline HDRS (SCORE) & $4.5(0.7)$ & $6.7(1.1)$ & $22.6(1.4)^{* *}$ \\
\hline HARS (SCORE) & $3.1(0.5)^{* *}$ & $17.3(1.5)$ & $19.5(1.8)$ \\
\hline GESTATION WEEKS & $34.4(3.6)$ & $37.0(3.4)^{*}$ & $33.1(5.5)$ \\
\hline GWK & CTRL & SA & $S A+S D$ \\
\hline RANGE & $\mathrm{N}(\%)$ & N (\%) & N (\%) \\
\hline $20-26$ & $0(0)$ & $0(0)$ & $4(6)$ \\
\hline $27-29$ & $6(15)$ & $10(13.9)$ & $7(10.5)$ \\
\hline $30-32$ & $8(22.5)$ & $6(8.3)$ & $9(13.4)$ \\
\hline $33-35$ & $11(27.5)$ & $8(11.1)$ & $12(17.9)$ \\
\hline $36-38$ & $12(30)$ & $17(23.6)$ & $14(20.9)$ \\
\hline $39-41$ & $3(7.5)$ & $31(43)$ & $21(31.4)$ \\
\hline Marital status & $\mathrm{N}(\%)$ & N (\%) & N (\%) \\
\hline Never Married & $9(23)$ & $10(14)$ & $9(13)$ \\
\hline Married & $4(10)$ & $19(26)$ & $23(34)$ \\
\hline Divorced & $12(30)$ & $5(4.1)$ & $4(3)$ \\
\hline Cohabiting & $15(38)$ & $38(56)$ & $31(52)$ \\
\hline Education level & $N(\%)$ & $\mathrm{N}(\%)$ & $\mathrm{N}(\%)$ \\
\hline Elementary school & $0(0)$ & $3(4.2)$ & $15(22)$ \\
\hline Middle school & $22(55)$ & $24(33)$ & $23(34)$ \\
\hline High school & $11(28)$ & $25(35)$ & $19(28)$ \\
\hline Bachelor's degree & $7(18)$ & $11(15)$ & $7(10)$ \\
\hline Postgraduate & $0(0)$ & $4(5.6)$ & $0(0)$ \\
\hline Technician degree & $0(0)$ & $5(6.9)$ & $3(4.5)$ \\
\hline Working status & $\mathrm{N}(\%)$ & $\mathrm{N}(\%)$ & $\mathrm{N}(\%)$ \\
\hline Employee & $9(23)$ & $10(14)$ & $8(12)$ \\
\hline Unemployed & $0(0)$ & $0(0)$ & $0(0)$ \\
\hline Home labor & $24(60)$ & $49(68)$ & $44(66)$ \\
\hline Commerce & $7(18)$ & $3(4.2)$ & $5(7.5)$ \\
\hline Profession & $0(0)$ & $5(6.9)$ & $3(4.5)$ \\
\hline Other & $0(0)$ & $5(6.9)$ & $7(10)$ \\
\hline
\end{tabular}

$\left(^{*}\right) p<0.005$, indicates the differences in age and gwks found among tested groups. $\left(^{* *}\right) p<0.001$, indicates the differences found between the final HDRS score values estimated for either the SA + SD and the CTRL groups, respectively; or between the final HARS score values estimated for each of the studied groups. Data are expressed as the mean \pm SD. Data were calculated using GraphPad-v.7

Abbreviations: $S A+S D$ severe anxiety plus comorbid severe depression; SA severe anxiety, CTRL control, HDRS Hamilton Depression Rating Scale, HARS Hamilton Anxiety Rating Scale, BMI Body Mass Index, GWK gestational weeks

correlations were found between age and Th-related cytokines. Significant differences were observed between the cytokines assayed and HARS (Tukey test, $p \leq 0.02$ ) and HDRS scores (Tukey test, $\mathrm{p} \leq 0.03$ ) in both the SA + SD and SA groups, respectively (data not shown).

\section{Th1:Th2 ratio}

Th1:Th2 ratios were estimated in each of the tested group. The estimated Th1:Th2 ratios (IFN- $\gamma$,-IL-4 and TNF- $\alpha$-IL4 ratios) in the control group were 0.5 and 0.6 respectively; whereas the Th1 (IFN-y,TNF- $\alpha$ ):Th2(IL-4) ratios estimated 


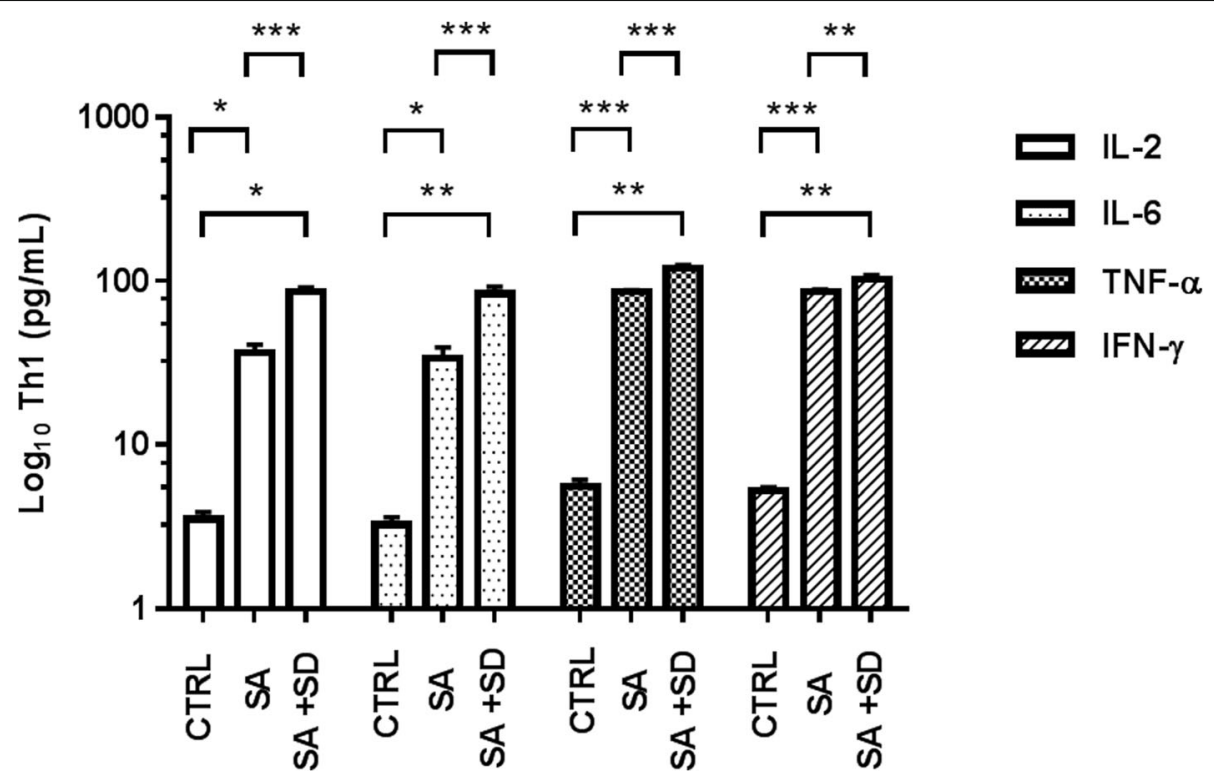

Fig. 1 Concentrations of Th1-related cytokines in tested groups. The figure depicts the Log $_{10}$ concentration values of estimated Th1-related cytokines in serum in the CTRL, SA, and SA + SD groups, respectively. Th1-related cytokines were quantified by flow cytometry (see methods). The concentration values of proinflammatory cytokines are expressed as the mean $\pm \mathrm{SEM}$, as described: CTRL; IL-2 $(3.5 \pm 0.4 \mathrm{pg} / \mathrm{mL}), \mathrm{IL}-6$ ( $3.3 \pm 0.4 \mathrm{pg} /$ $\mathrm{mL})$, TNF- $a(5.6 \pm 0.5 \mathrm{pg} / \mathrm{mL}), \mathrm{IFN}-\gamma(5.3 \pm 0.3 \mathrm{pg} / \mathrm{mL}) . \mathrm{SA} ; \mathrm{IL}-2(33.7 \pm 5.7 \mathrm{pg} / \mathrm{mL}), \mathrm{IL}-6(33.3 \pm 5.9 \mathrm{pg} / \mathrm{mL}), \mathrm{TNF}-\alpha(85.9 \pm 1.8 \mathrm{pg} / \mathrm{mL}), \mathrm{IFN}-\gamma(86.5 \pm 2.1$ $\mathrm{pg} / \mathrm{mL})$. SA + SD; IL-2 (85.8 $\pm 5.7 \mathrm{pg} / \mathrm{mL}), \mathrm{IL}-6(83.4 \pm 9.2 \mathrm{pg} / \mathrm{mL})$, TNF-a $(119.5 \pm 6.2 \mathrm{pg} / \mathrm{mL}), \mathrm{IFN}-\gamma(103.5 \pm 5.4 \mathrm{pg} / \mathrm{mL})$. The non-parametric, t-test analysis with Welch's correction was used to estimate the $p$-values for each Th2-related cytokine assayed among the studied groups. $(*) p \leq 0.05$; $\left.{ }^{* *}\right) ; p \leq 0.001 ;\left(^{* *}\right) p \leq 0.0001$. Significant differences were established at a $p<0.05$. Abbreviations: $\mathrm{SA}+\mathrm{SD}$, severe anxiety plus comorbid severe, severe anxiety; CTRL; control. Data were calculated using GraphPad v.7

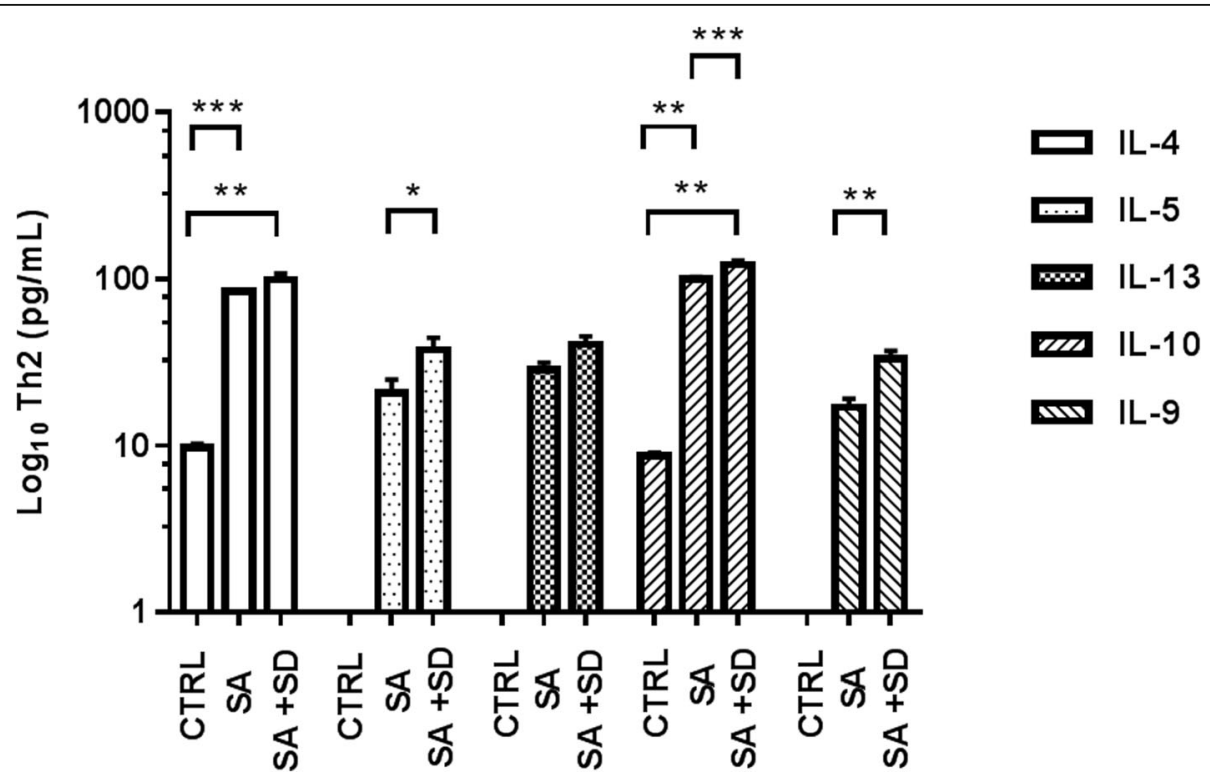

Fig. 2 Concentrations of Th2-related cytokines in the tested groups. The figure depicts the $\log _{10}$ concentration values of estimated Th2-related cytokines in serum in the CTRL, SA, and SA + SD groups, respectively. Th2-related cytokines were quantified by flow cytometry (see methods). The concentration values of anti-inflammatory cytokines are expressed as the mean \pm SEM, as described: CTRL; IL-4 ( $9.7 \pm 0.5 \mathrm{pg} / \mathrm{mL}), \mathrm{IL}-5, \mathrm{ND} ; \mathrm{IL}-13, \mathrm{ND} ; \mathrm{IL}-$ $10(8.7 \pm 0.4 \mathrm{pg} / \mathrm{mL}), I L-9, N D$. SA; IL-4 $(84.3 \pm 2.0 \mathrm{pg} / \mathrm{mL}), I L-5(20.7 \pm 4.2 \mathrm{pg} / \mathrm{mL}), I L-13(28.6 \pm 2.9 \mathrm{pg} / \mathrm{mL}), I L-10(99.8 \pm 2.7 \mathrm{pg} / \mathrm{mL}), \mathrm{L}-9$ ( $16.9 \pm 2.2 \mathrm{pg} / \mathrm{mL})$. $\mathrm{SA}+\mathrm{SD} ; \mathrm{IL}-4$ ( $99.1 \pm 8.2 \mathrm{pg} / \mathrm{mL}), \mathrm{IL}-5$ ( $37.5 \pm 6.5 \mathrm{pg} / \mathrm{mL}), \mathrm{LL}-13(40.3 \pm 4.9 \mathrm{pg} / \mathrm{mL}), \mathrm{LL}-10(121.4 \pm 7.9 \mathrm{pg} / \mathrm{mL}), I L-9(33.2 \pm 3.7 \mathrm{pg} / \mathrm{mL})$. The non-parametric, ttest analysis with Welch's correction was used to estimate the $p$ values for each Th2-related cytokine assayed among the studied groups. ( $\star$ ) nondetermined values; $\left(^{*}\right) p \leq 0.05 ;(* *) ; p \leq 0.001 ;(* * *) p \leq 0.0001$. Significant differences were established at a $p<0.05$. Abbreviations: ND, nondetermined; SA + SD, severe anxiety plus comorbid severe depression; SA, severe anxiety; CTRL; control. Data was calculated using GraphPad v.7 


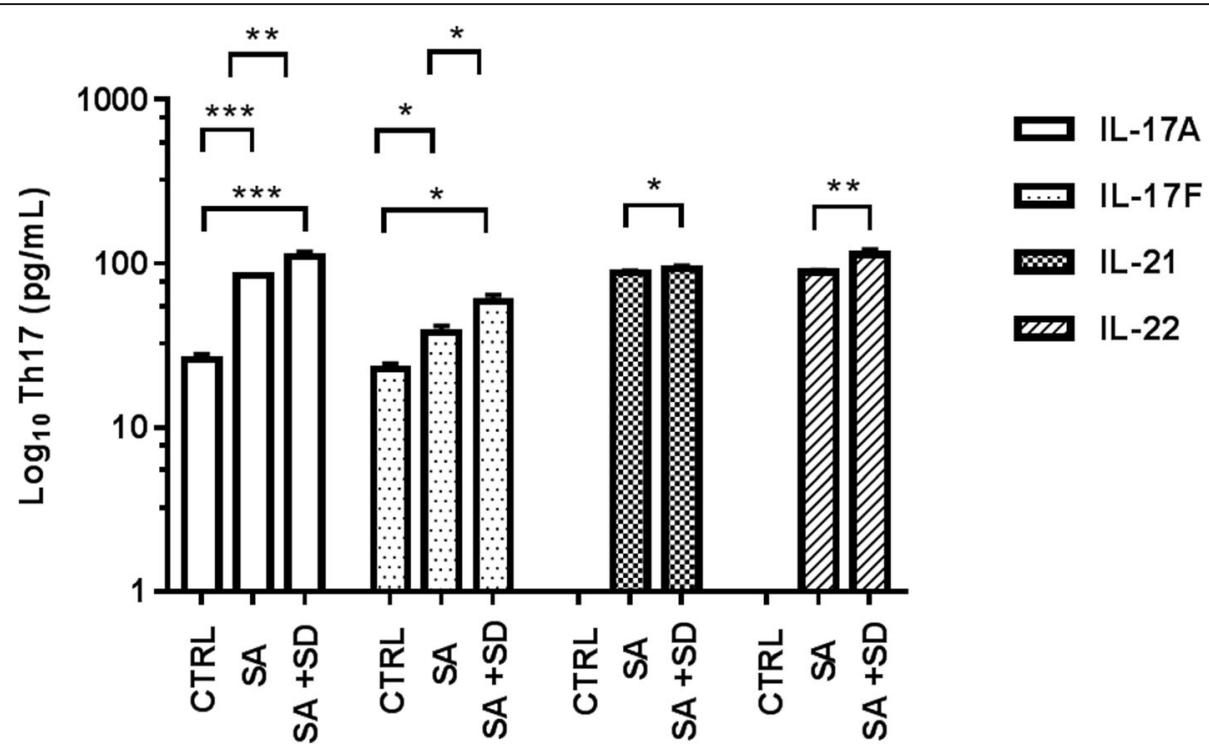

Fig. 3 Concentrations of Th17-related cytokines in tested groups. The figure depicts the Log 10 concentration values of estimated Th17-related cytokines in serum in the CTRL, SA, and SA + SD groups, respectively. Th17-related cytokines were quantified by flow cytometry (see methods). The concentration values of proinflammatory Th17-related cytokines are expressed as the mean \pm SEM, as described: CTRL; IL-17A (25.9 $\pm 2.1 \mathrm{pg} /$ $\mathrm{mL}) ; \mathrm{IL}-17 \mathrm{~F}(22.7 \pm 1.8 \mathrm{pg} / \mathrm{mL}) ; \mathrm{IL}-21, \mathrm{ND} ; \mathrm{IL}-22, \mathrm{ND}$. SA; IL-17A $(84.6 \pm 1.3 \mathrm{pg} / \mathrm{mL}), \mathrm{IL}-17 \mathrm{~F}(38.1 \pm 3.7 \mathrm{pg} / \mathrm{mL}), \mathrm{IL}-21(87.9 \pm 2.4 \mathrm{pg} / \mathrm{mL}), \mathrm{IL}-22(88.9 \pm 2.7$ $\mathrm{pg} / \mathrm{mL})$. SA + SD; IL-17A (109. $9 \pm 18.3 \mathrm{pg} / \mathrm{mL}), I L-17 F(59.1 \pm 5.5 \mathrm{pg} / \mathrm{mL}), \mathrm{IL}-21(92.6 \pm 4.5 \mathrm{pg} / \mathrm{mL}), \mathrm{LL}-22(113.5 \pm 8.6 \mathrm{pg} / \mathrm{mL})$. The non-parametric, ttest analysis with Welch's correction was used to estimate the $p$ values for each Th2-related cytokine assayed among the studied groups. ( $\star$ ) non-determined values; $\left(^{*}\right) p \leq 0.05 ;\left(^{* *}\right) ; p \leq 0.001 ;\left(^{* *}\right) p \leq 0.0001$. Significant differences were established at a $p<0.05$. Abbreviations: ND, nondetermined; SA + SD, severe anxiety plus comorbid severe depression; SA, severe anxiety; CTRL; control. Data were calculated using GraphPad v.7

in the SA + SD group were 1.3 and 1.4, respectively. The estimated Th1:Th2 ratio values in the SA group were 0.9 for both the IFN- $\gamma: I L-4$ and TNF- $\alpha$ :IL-4 ratios.

\section{General linear model}

A general linear model was constructed to assess the relationship between biological and sociodemographic variables (Table 4). We used all studied groups as the dependent variable and serum concentrations of cytokines as the independent variable. Both HDRS and HARS scores were included as covariables. The model showed that both HDRS and HARS were significantly associated with interleukin concentrations among the studied groups; whereas only IL- 6 and TNF- $\alpha$ concentrations were defined by the groups. The model explained $96.5 \%$ of the variance, and the model provided a good fit of the data (R-squared $=0.965$; adjusted R-Squared $=0.996$ ).

\section{Discussion}

Our study comprised pregnant participants $(n=179)$ who were mostly recruited during the 3rd trimester of pregnancy. Cytokine concentrations varied as pregnancy progresses. Pro-inflammatory cytokines tend to increase at the final weeks of pregnancy, while anti-inflammatory cytokines show an opposite profile [39]. Some authors have argued that Th1-related cytokines play a crucial role in subjects exhibiting both depression and anxiety during pregnancy. In line with this, several studies have extensively documented that psychosocial stress and depressive symptoms are associated with elevations of inflammatory biomarkers in non-pregnant humans and animals $[40,41]$. In a similar context, perceived stress with elevated stress scores positively correlate with high IL-6 and TNF- $\gamma$ serum levels and with low IL-10 levels in healthy subjects exhibiting normal pregnancies [42].

Similar studies conducted in pregnant women have shown significant correlations between depressive symptoms and Th1/Th2-related biomarkers (IL-6, TNF- $\alpha$, IL-10) at mid and late pregnancy $[17,29,42]$; in addition to the increased plasma levels of IL-1 $\beta$ and IFN- $\gamma$, prenatal stress, and anxiety symptoms observed in pregnant women exhibiting high levels of anxiety after undergoing elective cesarean [43].

Recent findings show that anxiety is associated with increased levels of interleukin-6 (IL-6) and associated with an increased risk for diseases with an inflammatory etiology [44]. In a similar context, anti-inflammatory cytokines could play distinct roles in mood-associated disorders, as suggested in recent studies that show a correlation between Th2-related IL-13/IL-9 cytokine concentrations and the depressive Edinburgh Postnatal Depression Scale (EPDS) score; whereas IL-13 and IL-10 cytokine concentrations correlated with anxiety symptoms in pregnant women during mid-pregnancy [30]. 
Table 2 SSPS software v.24.0 was used to determine the Pearson-bilateral correlations among tested groups

\begin{tabular}{|c|c|c|c|c|}
\hline CTRL & & & & \\
\hline IL-6 & gwk & & & \\
\hline CORR & $0.353^{*}$ & & & \\
\hline SIG. & 0.03 & & & \\
\hline TNF- $\alpha$ & weight & $B M I$ & & \\
\hline CORR & $0.463^{* *}$ & $0.413^{*}$ & & \\
\hline SIG. & 0.004 & 0.01 & & \\
\hline $\mathrm{IFN}-\gamma$ & HARS & weight & & \\
\hline CORR & $(-) 0.426^{*}$ & $0.360^{*}$ & & \\
\hline SIG. & 0.01 & 0.03 & & \\
\hline SA & & & & \\
\hline IL-2 & HDRS & $g w k$ & & \\
\hline CORR & $0.388^{*}$ & (-) $0.439 *$ & & \\
\hline SIG. & 0.04 & 0.02 & & \\
\hline IL-6 & HDRS & $g w k$ & & \\
\hline CORR & $0.353^{*}$ & 0.325 & & \\
\hline SIG. & 0.03 & 0.053 & & \\
\hline TNF- $\alpha$ & HDRS & $g w k$ & weight & BMI \\
\hline CORR & $0.413^{*}$ & (-) $0.491 * *$ & $0.495^{* *}$ & $0.440 * *$ \\
\hline SIG. & 0.01 & 0.009 & 0.002 & 0.007 \\
\hline IFN- $\gamma$ & $g w k$ & weight & & \\
\hline CORR & (-) $0.410^{*}$ & $0.338^{*}$ & & \\
\hline SIG. & 0.03 & 0.04 & & \\
\hline \multicolumn{5}{|l|}{$S A+S D$} \\
\hline IL-2 & HDRS & $g w k$ & weight & $B M I$ \\
\hline CORR & $0.780^{* *}$ & (-) $0.607^{* *}$ & (-) $0.496 * *$ & (-) $0.454^{* *}$ \\
\hline SIG. & 0.0001 & 0.0001 & 0.006 & 0.01 \\
\hline IL-6 & HDRS & gwk & & \\
\hline CORR & $0.652^{* *}$ & (-) $0.522 * *$ & & \\
\hline SIG. & 0.0001 & 0.001 & & \\
\hline TNF- $\alpha$ & HDRS & $g w k$ & height & weight \\
\hline CORR & $0.565 * *$ & $(-) 0.633 * *$ & (-) $0.395^{*}$ & (-) $0.426 * *$ \\
\hline SIG. & 0.001 & 0.0001 & 0.03 & 0.02 \\
\hline IFN- $\gamma$ & HARS & $g w k$ & $B M I$ & \\
\hline CORR & $0.419 *$ & $0.371^{*}$ & (-) 0.363 & \\
\hline SIG. & 0.02 & 0.05 & 0.05 & \\
\hline IL-4 & height & & & \\
\hline CORR & $0.318^{* *}$ & & & \\
\hline SIG. & 0.02 & & & \\
\hline IL-5 & height & & & \\
\hline CORR & $0.411 *$ & & & \\
\hline SIG. & 0.03 & & & \\
\hline IL-13 & HDRS & weight & $B M I$ & \\
\hline CORR & $0.407^{*}$ & (-) $0.434^{*}$ & $(-) 0.356$ & \\
\hline SIG. & 0.03 & 0.02 & 0.06 & \\
\hline IL-10 & HDRS & $g w k$ & & \\
\hline CORR & $0.467 *$ & (-) $0.633^{* *}$ & & \\
\hline SIG. & 0.01 & 0.0001 & & \\
\hline IL-9 & HDRS & height & & \\
\hline CORR & $0.468 *$ & $(-) 0.530 * *$ & & \\
\hline SIG. & 0.01 & 0.003 & & \\
\hline IL-17A & HDRS & gwk & $g w k$ & \\
\hline CORR & $0.509 * *$ & $0.396 *$ & $(-) 0.453 *$ & \\
\hline SIG. & 0.005 & 0.03 & 0.01 & \\
\hline IL-17F & $g w k$ & & & \\
\hline CORR & $(-) 0.422^{*}$ & & & \\
\hline SIG. & 0.02 & & & \\
\hline
\end{tabular}

Abbreviations: $S A+S D$ severe anxiety plus comorbid severe depression, $S A$ severe anxiety, CTRL control, HDRS Hamilton Depression Rating Scale, HARS Hamilton Anxiety Rating Scale, BMI Body Mass Index, GWK gestational weeks, Corr correlation, Sig significance. $\left(^{*}\right)$ Significant correlation at a $p$-value of 0.05 ; $(* *)$ Significant correlation at a $p$-value of 0.01
Table 3 SSPS software v.24.0 was used to determine the Pearson correlations between parameters among tested groups

\begin{tabular}{|c|c|c|c|}
\hline CTRL & & & \\
\hline IL-10 & $A G E$ & & \\
\hline CORR & -0.38 & & \\
\hline SIG. & $0.05^{*}$ & & \\
\hline IL-17A & $A G E$ & & \\
\hline CORR & -0.45 & & \\
\hline SIG. & $0.02 *$ & & \\
\hline SA & & & \\
\hline IL-17A & HARS & & \\
\hline CORR & 0.43 & & \\
\hline SIG. & $0.03 *$ & & \\
\hline$S A+S D$ & & & \\
\hline IL-2 & $A G E$ & HARS & HDRS \\
\hline CORR & -0.45 & 0.45 & 0.68 \\
\hline SIG. & $0.02 *$ & $0.02 *$ & $0.0001 * *$ \\
\hline IL-6 & HARS & HDRS & \\
\hline CORR & 0.43 & 0.48 & \\
\hline SIG. & $0.03 *$ & $0.01^{*}$ & \\
\hline IL-9 & HARS & HDRS & \\
\hline CORR & 0.43 & 0.4 & \\
\hline SIG. & $0.03 *$ & $0.05^{*}$ & \\
\hline IL-17A & $A G E$ & HARS & \\
\hline CORR & -0.52 & 0.43 & \\
\hline SIG. & $0.006 * *$ & $0.03^{*}$ & \\
\hline IL-17F & $A G E$ & HARS & \\
\hline CORR & -0.5 & 0.43 & \\
\hline SIG. & $0.009 * *$ & $0.03^{*}$ & \\
\hline
\end{tabular}

Correlations and $p$-values were obtained after controlling gestational weeks, used as the dependent variable for the analysis (see text for details) Abbreviations: $S A+S D$ severe anxiety plus comorbid severe depression, $S A$ severe anxiety, CTRL control, HDRS Hamilton Depression Rating Scale, HARS Hamilton Anxiety Rating Scale, GWK gestational weeks, Corr correlation, Sig significance. $\left(^{*}\right)$ Significant correlations at a $p$-value $\leq 0.05 ;\left(^{* *}\right)$ Significant correlation at a $\mathrm{p}$-value $\leq 0.01$

Interestingly, the levels of proinflammatory cytokines were higher in women exhibiting anxiety and comorbid depression with respect to pregnant women displaying only anxiety. Nonetheless, some studies showed opposite results, as shown for a single case study that reported that pro-inflammatory cytokine levels were unrelated with depression and anxiety symptoms [28].

Some authors have reported a correlation between higher levels of IL- 6 and TNF- $\alpha$ and depression scores (CES-D) in subjects displaying major depression, but not 
Table 4 Test between subject and effects

\begin{tabular}{l|r|r|r|r|r}
\hline \multicolumn{1}{c|}{ Origin } & $\begin{array}{r}\text { Type III sum of } \\
\text { squares }\end{array}$ & df & Mean Square & F & p \\
\hline Corrected model & $64.383^{3}$ & 64 & 1.006 & 320.895 & $0.000^{* *}$ \\
Intercept & 0.002 & 1 & 0.002 & 0.572 & 0.456 \\
GWKs & 0.004 & 1 & 0.004 & 1.307 & 0.263 \\
HDRS & 1.948 & 1 & 1.948 & 621.476 & $0.000^{* *}$ \\
HARS & 0.045 & 1 & 0.045 & 14.343 & $0.001^{* *}$ \\
IL-6 & 0.059 & 8 & 0.007 & 2.334 & 0.048 \\
IL-4 & 0.018 & 6 & 0.003 & .968 & 0.465 \\
TNF- $\alpha$ & 0.138 & 17 & 0.008 & 2.594 & $0.013^{*}$ \\
IL-6 * IL-4 & 0.000 & 0 & & & \\
IL-6 * TNF- $\alpha$ & 0.001 & 1 & 0.001 & 0.366 & 0.550 \\
IL-4 * TNF- $\alpha$ & 0.000 & 0 & & & \\
IL-6 * IL-4 * TNF- $\alpha$ & 0.000 & 0 & & & \\
Error & 0.085 & 27 & & & \\
Total & 143.000 & 92 & & & \\
Corrected Total & 64.467 & 91 & & & \\
\hline
\end{tabular}

Dependent variable: groups CTRL, SA, SA + SD. (a) R-squared $=0.965$ (Adjusted R-squared $=0.996$ )

Abbreviations: HDRS Hamilton Depression Rating Scale, HARS Hamilton Anxiety Rating Scale, GWKs gestational weeks. Significant differences were established at a $p$-value $\leq 0.05$. $\left.{ }^{*}\right)$ Significant differences at a $p$-value $\left.\leq 0.05 ;{ }^{* *}\right)$ Significant differences at a $p$-value $\leq 0.01$

with high scores for stress perception in Afro-American and Caucasian pregnant women [29]. Our results suggest that variations in cytokine concentrations are likely influenced by the intensity of depressive symptoms. We show that pregnant women exhibiting severe anxiety and comorbid depression displayed significant increases in serum levels of Th1 and Th17-related cytokines, which correlated with HDRS scores.

Furthermore, a recent study conducted by Osborne [45] showed that women exhibiting high levels of depressive and anxious symptoms in the third trimester of pregnancy showed a proinflammatory burst of Th1-related cytokines. Interestingly, these authors assessed serum cytokines at five points during pregnancy and observed differences over time [45].

Such observations led us to control our correlation analysis by gestational weeks. As shown, only IL-2, IL-6, IL-9, and IL-17A cytokines remained as relevant immune mediators that significantly correlated with the HDRS score in the SA + SD group. Such observations suggest that regardless of the gestational week period, depressive symptoms appear to modify the cytokine profile, showing that higher levels of depressive symptoms are related to increased levels of proinflammatory cytokines. Moreover, the observed increases in both IFN- $\gamma$ : IL-4 and TNF- $\alpha$ : IL-4 ratios in the $\mathrm{SA}$ and $\mathrm{SA}+\mathrm{SD}$ groups suggest that an inflammatory process takes place in vulnerable subjects with severe anxiety, becoming conspicuous as depression emerges in women displaying severe depression.
Interestingly, using the general linear model of analysis of variance constructed to explain the interaction between variables among the studied groups, we observed that the rating scores obtained from both depression (HDRS) and anxiety (HARS) instruments applied to the participants were associated with interleukins' concentrations. However, only IL- 6 and TNF- $\alpha$ cytokines were identified as relevant pro-inflammatory biomarkers that could differentiate between the groups exhibiting affective symptoms (depressive versus the anxiety/non-depressive group) and the control group.

Both IL-2 and IL-6 are well recognized as pro-inflammatory cytokines, while IL-9 is considered a pleiotropic cytokine that has direct and indirect effects on multiple cell types that affect immunity and inflammation [46].

Based on the high degree of plasticity and differentiation of Th17 cell lines into Th1 or Treg cells [47], it might be feasible to posit that such Th17-related cytokines could elicit qualitatively distinct responses and interactions with the brain and placenta, promoting the expression of depressive and anxiety symptoms in vulnerable women at high risk of developing mood disorders [5].

Finally, the activation of specific cytokine-associated signaling pathways in the brain and placenta has been proposed as an immune-related mechanism that appears to play a crucial role in triggering and/or exacerbating depressive symptoms during pregnancy, enhancing negative perinatal outcomes [5, 16-18, 24-26]. 


\section{Conclusions and perspectives}

Our data show that patients exhibiting severe anxiety and comorbid depression display significant increases in serum proinflammatory and anti-inflammatory-related cytokines that correlated with the intensity of depressive symptoms measured by HDRS. Furthermore, the present results support much of interacting network systems driven by the immune and neuroendocrine systems and placenta $[5-8,18,19]$, and whose dysregulation might precipitate or exacerbate depressive symptoms in vulnerable women exhibiting severe anxiety during pregnancy.

Our results parallel other studies that have shown that Th1 cytokines appear to modulate anxious symptoms in non-pregnant populations with increased levels of Th1 cytokines and higher ratios in the Th1:Th2 immune balance [48]. Most of our correlations found between depression and anxiety scores with $\mathrm{Th}(1), \mathrm{Th}(2)$, and $\mathrm{Th}(17)$-related cytokines support the role of inflammatory cytokines in mood-associated disorders during pregnancy.

As anxiety and depression are usually comorbid, it is imperative to perform studies to explore further correlations between biological mediators and anxiety symptoms in women at high risk of developing severe depression during mid to late pregnancy. It is worth noting that the emergence of depression associated with an immune-related inflammatory process and expression of a Th1-cytokine profile during pregnancy should be taken seriously. Activation of the innate immune system and their immune mediators might lead to a chronic inflammatory process that, if not controlled, might precipitate in both negative obstetric and neonatal outcomes [5]. Thus, our results show that depressive symptoms, regardless of anxiety and gestational weeks, are highly related to the increase of blood proinflammatory cytokines.

\section{Limitations}

Several limitations in the present study should be noted. First, we applied the self-reported HARS and HDRS instruments used to evaluate depressive and anxiety symptoms, which have not been extensively used in pregnant women, as compared to other psychological instruments (e.g., the CES-D, EPDS, STAI, and GAD-7). Second, blood sampling was performed at a single time (T1) at the entry of participants into the study. Thus, to detect changes in cytokine concentration during late pregnancy two specific blood-sampling time-points (T1, T2) must have been elected as the optimum (i.e., at 28 and 39 gwk) in our studied population. Third, we did not evaluate the prevalence of cigarette smoking in recruited participants, an important variable that might have influenced our results. Also, pre-gestational weight could contribute to further information about the relevance of weight gain and BMI associated with the determination of a Th1, Th2 cytokine profile.

\section{Abbreviations}

CES-D: Center for Epidemiological Studies Depression Inventory; CTRL: control; DHEA-S: Dehydroepiandrosterone sulfate; DSM-5: Diagnostic and Statistical Manual of Mental Disorders; EPDS: Edinburgh Perinatal Depression Scale; GAD-7: Generalized Anxiety Disorder Screener; GWK: gestation weeks; HARS: Hamilton Anxiety Rating Scale; HDRS: Hamilton Depression Rating Scale; IFN: interferon; IL: interleukins; MDD: major depressive disorder; SA: severe anxiety; SD: severe depression; SSRI: selective serotonin reuptake inhibitors; STAI: state anxiety subscale of the state-trait anxiety inventory; TNF: Tumor Necrosis Factor

\section{Acknowledgments}

This study was supported by the following grants: FOSSIS/CONACyT Project No. 272458, and INPer Project No. 212250-3000-10916-01-16. We thank B.Sc. Irma Pedroza for her unconditional support during the evaluation of the psychological rating scales. Also, we gratefully thank Dr. Coronel and Dr. Takashima for providing the GO-outpatient unit used to interview and obtain the blood samples from pregnant women. We also thank Dr. Cruz and Dr. Hernández-Ruiz for providing their lab-units and equipment used for serum separation and cryopreservation of vials containing serum samples.

\section{Funding}

The present studied was funded by FOSSIS/CONACyT Grant No. 272458 and the INPer Grant No. 212250-3000-10916-01-16. The funding institutions had no participation in the design of the study and collection, analysis, and interpretation of data, nor in the elaboration of the present manuscript.

\section{Availability of data and materials}

The datasets used and/or analyzed during the current study are available from the corresponding author on reasonable request.

\section{Authors' contributions}

LGP and FRM designed and conducted the study. LGP, FRM, and CAI wrote the body of the manuscript. GMS and CAI made major contributions to the writing and editing of several parts of the manuscript. MHI measured and analyzed the serum concentration of cytokines assayed by flow cytometry. LGP and FLB contributed to the statistical analysis of the manuscript. CCFM and SMF conducted the interview and recruitment of pregnant women after a psychiatric evaluation of patients. FRM conducted the psychiatric intervention of participants and evaluated the scores obtained from applied instruments. Both SMF and COD rated the depressive and anxiety scales used to assess the intensity of affective symptoms in patients recruited to the study. SMF and FLB conducted the blood sampling. LGP and SAF collected the fresh blood samples from the GO department before labprocessing of samples. LGP, CFC, HRJ, and PMA, conducted the labprocessing of fresh blood samples (centrifuge and separation of serum samples into vials, storage of serum vials). LGP, SMF, MHI, HRJ, CFC, and PMA collected both biochemical and clinical data from participants recruited into the study. All authors read and approved the final manuscript.

Ethics approval and consent to participate

Both the Ethics and Research Committees from the General Hospital of Mexico approved the human study and gave their permission to involve human participants. All participants provided a signed written informed consent before their recruitment to the study.

\section{Consent for publication}

Not applicable.

\section{Competing interests}

The authors declare that they have no competing interests in any issue concerning with the publication of the present manuscript.

\section{Publisher's Note}

Springer Nature remains neutral with regard to jurisdictional claims in published maps and institutional affiliations.

\section{Author details}

${ }^{1}$ Instituto Nacional de Pernatologia, Isidro Espinosa de los Reyes, Montes Urales \# 800, Col Lomas de Virreyes, 11000 (Mexico City), CD MX, Mexico. ${ }^{2}$ Instituto Nacional de Psiquiatría, 14370 CD MX, (Mexico City), Mexico. 
${ }^{3}$ Hospital General de México, Dr. Eduardo Liceaga, 06720 (Mexico City), CD MX, Mexico. ${ }^{4}$ HIPAM-Unidad de Investigación en Medicina-UNAM, Hospital General de México Dr. Eduardo Liceaga, 06720 (Mexico City), CD MX, Mexico. ${ }^{5}$ Unidad de Investigación en Reproducción Humana, Instituto Nacional de Perinatología-Facultad de Química, Universidad Nacional Autónoma de México, 04510 (Mexico City), CD MX, Mexico. ${ }^{6}$ Consejo Nacional de Ciencia y Tecnología/CONACyT, 03940 (Mexico City), CD MX, Mexico.

\section{Received: 2 August 2018 Accepted: 21 March 2019}

\section{Published online: 03 April 2019}

\section{References}

1. Dowlati Y, et al. A meta-analysis of cytokines in major depression. Biol Psychiatry. 2010;67(5):446-57.

2. Kopschina Feltes $P$, et al. Anti-inflammatory treatment for major depressive disorder: implications for patients with an elevated immune profile and non-responders to standard antidepressant therapy. J Psychopharmacol. 2017;31(9):1149-65.

3. Miller AH, Maletic $V$, Raison CL. Inflammation and its discontents: the role of cytokines in the pathophysiology of major depression. Biol Psychiatry. 2009; 65(9):732-41.

4. Rush AJ, et al. Sequenced treatment alternatives to relieve depression (STAR*D): rationale and design. Control Clin Trials. 2004;25(1):119-42.

5. Leff-Gelman $P$, et al. The immune system and the role of inflammation in perinatal depression. Neurosci Bull. 2016;32(4):398-420.

6. Cheng $C Y$, Pickler RH. Perinatal stress, fatigue, depressive symptoms, and immune modulation in late pregnancy and one month postpartum. ScientificWorldJournal. 2014;2014:652630.

7. Marcus SM, et al. Depressive symptoms among pregnant women screened in obstetrics settings. J Women's Health (Larchmt). 2003;12(4):373-80.

8. Bittner $\mathrm{A}$, et al. Early intervention in pregnant women with elevated anxiety and depressive symptoms: efficacy of a cognitive-behavioral group program. J Perinat Neonatal Nurs. 2014;28(3):185-95.

9. Stuart-Parrigon K, Stuart S. Perinatal depression: an update and overview. Curr Psychiatry Rep. 2014;16(9):468.

10. Dayan J, et al. Prenatal depression, prenatal anxiety, and spontaneous preterm birth: a prospective cohort study among women with early and regular care. Psychosom Med. 2006;68(6):938-46.

11. Pakenham Kl, Smith A, Rattan SL. Application of a stress and coping mode to antenatal depressive symptomatology. Psychol Health Med. 2007;12(3): 266-77.

12. Fairbrother $\mathrm{N}$, et al. Psychometric evaluation of the multidimensional assessment of fatigue scale for use with pregnant and postpartum women. Psychol Assess. 2008;20(2):150-8.

13. Chien $L Y$, Ko YL. Fatigue during pregnancy predicts caesarean deliveries. J Adv Nurs. 2004;45(5):487-94.

14. Field T, Diego M, Hernandez-Reif M. Prenatal depression effects on the fetus and newborn: a review. Infant Behav Dev. 2006:29(3):445-55.

15. Piccinni MP. T cell tolerance towards the fetal allograft. J Reprod Immunol. 2010;85(1):71-5.

16. Meltzer-Brody S. New insights into perinatal depression: pathogenesis and treatment during pregnancy and postpartum. Dialogues Clin Neurosci. 2011;13(1):89-100

17. Saito $\mathrm{S}$, et al. Th1/Th2/Th17 and regulatory T-cell paradigm in pregnancy. Am J Reprod Immunol. 2010;63(6):601-10.

18. Mastorakos G, Ilias I. Maternal and fetal hypothalamic-pituitary-adrenal axe during pregnancy and postpartum. Ann N Y Acad Sci. 2003;997:136-49.

19. Rosenblat JD, et al. Inflamed moods: a review of the interactions between inflammation and mood disorders. Prog Neuro-Psychopharmacol Biol Psychiatry. 2014;53:23-34.

20. Wohleb ES, et al. Peripheral innate immune challenge exaggerated microglia activation, increased the number of inflammatory CNS macrophages, and prolonged social withdrawal in socially defeated mice. Psychoneuroendocrinology. 2012;37(9):1491-505.

21. Wohleb ES, et al. Beta-adrenergic receptor antagonism prevents anxiety-like behavior and microglial reactivity induced by repeated social defeat. J Neurosci. 2011;31(17):6277-88.

22. Czeh M, Gressens P, Kaindl AM. The yin and yang of microglia. Dev Neurosci. 2011;33(3-4):199-209.

23. Venneti S, Lopresti BJ, Wiley CA. Molecular imaging of microglia/ macrophages in the brain. Glia. 2013;61(1):10-23.
24. Glynn LM, et al. Pattern of perceived stress and anxiety in pregnancy predicts preterm birth. Health Psychol. 2008;27(1):43.

25. Hobel CJ, Goldstein A, Barrett ES. Psychosocial stress and pregnancy outcome. Clin Obstet Gynecol. 2008;51(2):333-48.

26. Latendresse $\mathrm{G}$. The interaction between chronic stress and pregnancy: preterm birth from a biobehavioral perspective. J Midwifery Womens Health. 2009:54(1):8-17.

27. Latendresse G, Ruiz RJ, Wong B. Psychological distress and SSRI use predict variation in inflammatory cytokines during pregnancy. Open J Obstet Gynecol. 2013;3(1A):184.

28. Christian LM, et al. Depressive symptoms are associated with elevated serum proinflammatory cytokines among pregnant women. Brain Behav Immun. 2009;23(6):750-4.

29. Simpson W, et al. Relationship between inflammatory biomarkers and depressive symptoms during late pregnancy and the early postpartum period: a longitudinal study. Rev Bras Psiquiatr. 2016;38(3):190-6.

30. Karlsson $L$, et al. Cytokine profile and maternal depression and anxiety symptoms in mid-pregnancy - the FinnBrain birth cohort study. Arch Womens Ment Health. 2017;20(1):39-48.

31. Dantzer $\mathrm{R}$, et al. From inflammation to sickness and depression: when the immune system subjugates the brain. Nat Rev Neurosci. 2008;9(1):46.

32. O'Donovan A, et al. Clinical anxiety, cortisol and interleukin-6: evidence for specificity in emotion-biology relationships. Brain Behav Immun. 2010;24(7): 1074-7.

33. Field T. Prenatal anxiety effects: a review. Infant Behav Dev. 2017;49:120-8.

34. Hamilton M. The assessment of anxiety states by rating. Br J Med Psychol. 1959;32(1):50-5

35. Hamilton M. A rating scale for depression. J Neurol Neurosurg Psychiatry. 1960;23:56-62.

36. Ramos-Brieva JA, Cordero-Villafafila A. A new validation of the Hamilton rating scale for depression. J Psychiatr Res. 1988;22(1):21-8.

37. Lobo A, et al. Validation of the Spanish versions of the Montgomery-Asberg depression and Hamilton anxiety rating scales. Med Clin (Barc). 2002;118(13): 493-9.

38. Maier W, et al. The Hamilton anxiety scale: reliability, validity and sensitivity to change in anxiety and depressive disorders. J Affect Disord. 1988;14(1): $61-8$.

39. Snegovskikh V, Park JS, Norwitz ER. Endocrinology of parturition. Endocrinol Metab Clin N Am. 2006:35(1):173-91 viii.

40. Coussons-Read ME, Okun ML, Nettles CD. Psychosocial stress increases inflammatory markers and alters cytokine production across pregnancy. Brain Behav Immun. 2007:21(3):343-50.

41. Raison CL. Inflammatory depression: a trifecta of trouble. J Clin Psychiatry. 2014:75(6):663-4.

42. Coussons-Read ME, et al. Prenatal stress alters cytokine levels in a manner that may endanger human pregnancy. Psychosom Med. 2005:67(4):625-31.

43. Hu Y, Huang K. Placenta response of inflammation and oxidative stress in low-risk term childbirth: the implication of delivery mode. BMC Pregnancy and Childbirth. 2017;17(1):407.

44. Baker DG, Nievergelt CM, O'Connor DT. Biomarkers of PTSD: neuropeptides and immune signaling. Neuropharmacology. 2012;62(2):663-73.

45. Osborne $L M$, et al. Innate immune activation and depressive and anxious symptoms across the peripartum: an exploratory study. Psychoneuroendocrinology. 2019;99:80-6.

46. Goswami R, Kaplan MH. A brief history of IL-9.J Immunol. 2011:186(6):3283-8.

47. Guéry L, Hugues S. Th17 cell plasticity and functions in cancer immunity. Biomed Res Int. 2015:2015.

48. Hou R, et al. Peripheral inflammatory cytokines and immune balance in generalised anxiety disorder: case-controlled study. Brain Behav Immun. 2017:62:212-8 